\title{
CONSUMO DE OXIGÊNIO NO DOMÍNIO DE INTENSIDADE SEVERO DURANTE TESTE INCREMENTAL E RETANGULAR
}

\author{
OXYGEN CONSUMPTION IN THE SEVERE EXERCISE INTENSITY DOMAIN \\ DURING INCREMENTAL AND SQUARE-WAVE TEST MODES
}

\begin{abstract}
RESUMO
Testes incrementais são comumente utilizados para predizer respostas metabólicas durante exercício retangular, porém, em alguns casos, os resultados preditos e observados podem não ser semelhantes. Objetivo principal: Verificar a hipótese de que $\mathrm{OVO}_{2}$ determinado em algumas intensidades do domínio severo, durante teste incremental, é menor do que os valores observados em testes retangulares. Procedimentos metodológicos: Oito homens fisicamente ativos (21,5 \pm 4 anos, $73,7 \pm 5,3 \mathrm{~kg}$ e 180,8 $\pm 4,1 \mathrm{~cm}$ ) realizaram: 1) teste incremental em cicloergômetro para determinação do $\mathrm{VO}_{2}$ das intensidades $\left(\mathrm{VO}_{2 \mathrm{NC}}\right)$; 2) testes retangulares em três intensidades severas (Leve $(\mathrm{L})$, Média $(\mathrm{M})$ e Pesada $\left.(\mathrm{P})\right)$ para comparar $\circ \mathrm{VO}_{2}\left(\mathrm{VO}_{2 \mathrm{RET}}\right)$ observado com o $\mathrm{VO}_{2 \mathrm{NC}}$ correspondente, adicionalmente, determinar a potência crítica (PCrit); 3 ) teste retangular na intensidade da PCrit para comparar a resposta temporal do $\mathrm{VO}_{2}$ com $\mathrm{P}$, M e L em quatro momentos. Resultados: $O \mathrm{VO}_{2 \mathrm{NC}}$, na intensidade $\mathrm{L}$, subestimou o $\mathrm{VO}_{2 \mathrm{RET}}\left(3,2 \pm 0,5 \mathrm{vs} .3,8 \pm 0,6 \mathrm{I} . \mathrm{min}^{-1}\right)$, não houve diferença nas outras intensidades (M: 3,5 $\pm 0,5$ vs. 3,6 $\pm 0,5 \mathrm{I} \cdot \mathrm{min}^{-1}$; P: $3,7 \pm 0,5$ vs. 3,4 $\pm 0,6 \mathrm{I} \cdot \mathrm{min}^{-1}$; PCrit: $2,7 \pm 0,5$ vs. $3,0 \pm 0,6 \mathrm{I} \cdot \mathrm{min}^{-1}$ ). O VO na PCrit foi menor em todos os momentos, apresentou estabilização e não atingiu o $\mathrm{VO}_{2 \max }$. Inicialmente, na intensidade $\mathrm{L}$, o $\mathrm{VO}_{2}$ foi menor do que seus pares. Contudo, no momento final, atingiu o $\mathrm{VO}_{2 \max }$ e se igualou às outras intensidades severas. Conclusão: $\mathrm{O}$ teste incremental subestima o $\mathrm{VO}_{2}$ observado em testes retangulares para intensidades mais leves do domínio severo.
\end{abstract}

Palavras-chave: Captação de oxigênio; Domínio Severo; Carga constante; Teste progressivo.

\section{ABSTRACT}

Incremental tests are commonly used to predict metabolic responses during square-wave mode exercises. However, in some cases the predicted and observed results may be not similar. Main objective: To verify the hypothesis that, at certain intensities in the severe domain, $\mathrm{VO}_{2}$ determined during the incremental test is lower than that observed at these workloads using the square-wave test. Methodological proceedings: Eight physically active males ( $21.5 \pm 4$ years, 73.7 $\pm 5.3 \mathrm{~kg}$ and $180.8 \pm 4.1 \mathrm{~cm}$ ) performed: 1$)$ an incremental test on cycle ergometer to determine the $\mathrm{VO}_{2}$ of the intensities $\left.\left(\mathrm{VO}_{2 \mathrm{INC}}\right) ; 2\right)$ a square-wave test at three severe intensities (Light $(\mathrm{L})$, Medium $(\mathrm{M})$ and Heavy $(\mathrm{H})$ ) to determine the critical power (CP) and to compare the $\mathrm{VO}_{2}\left(\mathrm{VO}_{2 \mathrm{RET}}\right)$ observed with the $\left.\mathrm{VO}_{2 \mathrm{INC}} ; 3\right)$ a square-wave test at $\mathrm{CP}$ intensity to compare the time response of $\mathrm{VO}_{2}$ with $\mathrm{P}, \mathrm{M}$ and $\mathrm{L}$, at four different sample times. Results: The $\mathrm{VO}_{2 \mathrm{NNC}}$ at $\mathrm{L}$ intensity underestimated the $\mathrm{VO}_{2 \mathrm{RET}}\left(3.2 \pm 0.5 \mathrm{vs}\right.$. $\left.3.8 \pm 0.6 \mathrm{I} . \mathrm{min}^{-1}\right)$. No differences were observed at the other intensities (M: $3.5 \pm 0.5 \mathrm{vs}$. $3.6 \pm 0.5$ I. $\mathrm{min}^{-1} ; \mathrm{H}: 3.7 \pm 0.5$ vs. $3.4 \pm 0.6 \mathrm{I} . \mathrm{min}^{-1} ; \mathrm{CP}: 2.7 \pm 0.5$ vs. $\left.3.0 \pm 0.6 \mathrm{I} . \mathrm{min}^{-1}\right)$. The $\mathrm{VO}_{2}$ at $\mathrm{CP}$ was lower at all times, exhibited a steady state and did not achieve $\mathrm{VO}_{2 \max }$. Initially, at $\mathrm{L}$ intensity, the $\mathrm{VO}_{2}$ was smaller than its pairs. However, at the last sample time, it achieved $\mathrm{VO}_{2 \max }$ and became equal to the other severe intensities. Conclusion: The incremental test underestimates the $\mathrm{VO}_{2}$ observed at lighter intensities within the severe domain.

Key words: Oxygen uptake; Severe domain; Constant power; Progressive test.

1 Universidade Estadual Paulista "Julio de Mesquita Filho" - campus de Rio Claro. Brasil. Instituto de Biociências - Departamento de Educação Física 2 Núcleo de Atividade Física Esporte e Saúde - NAFES 


\section{INTRODUÇÃO}

Os testes incrementais possibilitam a determinação de importantes parâmetros aeróbios, como o limiar anaeróbio ${ }^{1}$ e o consumo máximo de oxigênio $\left(\mathrm{VO}_{2 \max }\right)^{2}$. Adicionalmente, podem ser usados para observar a demanda de $\mathrm{O}_{2}$ em intensidades abaixo do limiar anaeróbio ${ }^{3}$

Durante um teste incremental máximo, as cargas de trabalho aplicadas ao longo do tempo produzem uma resposta linear do $\mathrm{VO}_{2}$. Admite-se que, para uma mesma intensidade, esse teste é capaz de refletir o $\mathrm{VO}_{2}$ observado em exercícios com carga constante (retangular).

Porém, a resposta do $\mathrm{VO}_{2}$ em exercícios retangulares apresenta características peculiares, as quais dependem da intensidade e duração da tarefa, conforme o domínio do exercício ${ }^{4-8}$. Exercícios realizados no domínio moderado (i.e. abaixo do limiar de lactato) levam à estabilização do $\mathrm{VO}_{2} \mathrm{em}$ valores inferiores ao do $\mathrm{VO}_{2 \max }$. No domínio intenso, compreendido entre o limiar de lactato e o máximo estado estável de lactato, o $\mathrm{VO}_{2}$ apresenta uma estabilização tardia, porém sem atingir o $\mathrm{VO}_{2 \max }^{4,9}$. A característica principal no domínio severo é a ausência de estabilização e a tendência a atingir o $\mathrm{VO}_{2 \max }{ }^{4}$.

Em testes incrementais, a imposição de diferentes intensidades de exercício é realizada em intervalos de tempo geralmente pré-fixados. Isso promove a passagem pelos três domínios de intensidade e possibilita medições fisiológicas em todas as cargas. Nesse tipo de teste o $\mathrm{VO}_{2 \max }$ só é alcançado na última ${ }^{2}$, ou duas últimas cargas de trabalho ${ }^{10}$, as quais são mais elevadas do que a intensidade correspondente ao máximo estado estável de lactato. Entretanto, a partir de dados de exercícios retangulares, poder-seia prever que, todo e qualquer exercício realizado no domínio severo deveria levar ao $\mathrm{VO}_{2 \max }{ }^{5}$, o que de fato parece não encontrar semelhança com a resposta observada, durante testes incrementais, em algumas intensidades severas.

A relação entre intensidades do domínio severo e o tempo decorrido até a exaustão (tlim) se ajusta a uma hipérbole retangular ${ }^{4,11}$. A intensidade correspondente à assíntota, denominada de potência crítica (PCrit) ${ }^{11}$, é descrita como a mais alta intensidade de exercício retangular na qual o $\mathrm{VO}_{2 \max }$ não é atingido ${ }^{12}$. Em outras palavras, pode-se levantar a hipótese de que, durante um teste incremental, o $\mathrm{VO}_{2}$ medido nas primeiras intensidades dentro do domínio severo é subestimado. A investigação dessa resposta metabólica é importante para verificar a existência de limitações na predição do $\mathrm{VO}_{2}$ para cargas retangulares severas, realizada através de testes incrementais.

Portanto, o objetivo do presente estudo foi o de explorar e comparar os valores de $\mathrm{VO}_{2}$ observados em testes incrementais com exercícios retangulares, realizados no domínio severo de exercício, em particular, para testar a hipótese de que nas intensidades mais leves, utilizadas para a predição da $\mathrm{PCrit}, \mathrm{o} \mathrm{VO}_{2}$ observado por meio de teste incremental subestima o valor real determinado em teste retangular.

\section{PROCEDIMENTOS METODOLÓGICOS}

Participaram do estudo oito jovens do gênero masculino, saudáveis e fisicamente ativos, com 21,5 \pm 4 anos de idade, $73,7 \pm 5,3 \mathrm{~kg}$ de massa corporal e 180,8 $\pm 4,1 \mathrm{~cm}$ de estatura. $O$ termo de consentimento livre e esclarecido foi assinado após terem sido informados dos objetivos, riscos e benefícios envolvidos no testes, bem como da liberdade de abandonar os testes a qualquer momento. Os procedimentos foram aprovados pelo Comitê de Ética em Pesquisa da UNESP - Rio Claro.

Durante quatro dias, os participantes foram conduzidos ao laboratório e submetidos, inicialmente, a um teste incremental para a determinação do $\mathrm{VO}_{2 \max }$. Outros três exercícios retangulares de caráter exaustivo e com intensidades severas, preconizados para a determinação da PCrit, foram distribuídos e realizados nos dias subseqüentes. Adicionalmente, cinco dos oito participantes realizaram uma quinta visita ao laboratório para um teste retangular não exaustivo, na intensidade da PCrit.

Todos os testes foram realizados em um cicloergômetro de frenagem mecânica (Monarck ${ }^{\circledR}$ ). A coleta das trocas gasosas foi realizada através do aparelho de espirometria, modelo $\mathrm{VO} 2000^{\circledR}$ (MedGraphics - St. Paul MN, USA). O aparelho foi ajustado para o método de coletas a cada três ciclos respiratórios e operado via computador pelo software Aerograph ${ }^{\circledR}$. A calibração foi realizada antes de cada coleta, utilizando-se volume (3 litros) e concentrações conhecidas de $\mathrm{O}_{2}(17 \%)$ e $\mathrm{CO}_{2}(5 \%)$.

Previamente ao início de todos os testes, os participantes realizaram 5 minutos de aquecimento, com carga correspondente a $60 \mathrm{~W}$, seguido de 5 minutos de intervalo, no qual, o participante tinha a liberdade de fazer alongamentos, de acordo com seus hábitos de atividade física. Após esse período foram encaminhados ao ergômetro e submetidos aos procedimentos para início da coleta do $\mathrm{VO}_{2}$.

Os valores de $\mathrm{VO}_{2}$ medidos nos testes retangulares e incremental foram interpolados, linearmente, a cada 1 segundo. Os valores máximos de $\mathrm{VO}_{2}$ encontrados no modo incremental $\left(\mathrm{VO}_{2 \text { max-INC }}\right)$ ou retangular $\left(\mathrm{VO}_{2 \max -\mathrm{RET} T}\right)$ foram definidos como a média dos valores encontrados nos últimos 30 segundos de exercício.

\section{Teste incremental}

A potência inicial do teste incremental correspondeu a uma intensidade de $20 \mathrm{~W}$ e acréscimos de $20 \mathrm{~W}$ a cada minuto. Os incrementos na carga de trabalho foram aplicados até o indivíduo não ser mais capaz de manter a potência requerida.

Para calcular o $\mathrm{VO}_{2}$ associado a cada estágio completado $\left(\mathrm{VO}_{2 \mathrm{INC}}\right)$, utilizou-se a média dos últimos 30 segundos de exercício, imediatamente anteriores, ao próximo incremento de carga. Para as intensidades com valores, em watts, não múltiplos de 20 (i.e.: $210,230 \mathrm{~W}$ ), adotou-se interpolação linear, através do $\mathrm{VO}_{2 \mathrm{INC}}$ determinado para as cargas de trabalho imediatamente abaixo e acima. Entretanto, algumas cargas aplicadas nos testes retangulares foram maiores do que a mais alta intensidade encontrada no teste 
incremental (Ppico). Para a determinação do $\mathrm{VO}_{2 \mathrm{NNC}}$ dessas intensidades, foi assumido o pressuposto de que acima da Ppico o consumo seria igual ao $\mathrm{VO}_{2 \text { max-INC' }}$, independente do valor absoluto da carga ${ }^{2}$.

Os dados do teste incremental foram também utilizados para a determinação da intensidade correspondente ao ponto de compensação respiratória, definida como a intensidade na qual houve aumento concomitante do

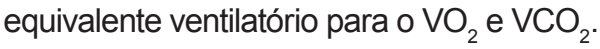

\section{Testes retangulares e determinação da PCrit}

Os testes retangulares foram realizados, respeitando um intervalo mínimo de 24 horas entre cada sessão. A intensidade severa aplicada em cada teste foi classificada de acordo com o tempo necessário para provocar a fadiga, da seguinte maneira: 60 - $200 \mathrm{~s}$, pesada (P); 200 - 400 s, média (M); e 400 - 600 s, leve (L). A fadiga foi caracterizada pela interrupção voluntária ou a impossibilidade de manutenção da intensidade estabelecida, apesar de encorajamento verbal.

Para determinar $\mathrm{O} \mathrm{VO}_{2}$ das intensidades $\mathrm{P}, \mathrm{M}$ e $\mathrm{L}\left(\mathrm{VO}_{2 \mathrm{RET}}\right)$ foram calculadas as médias dos valores observados nos últimos 30 segundos de exercício. Os valores de $\mathrm{VO}_{2 \mathrm{NCC}}$ foram comparados com os valores de $\mathrm{VO}_{2 \mathrm{RET}}$ para verificar a capacidade do teste incremental de predizer o consumo observado nos testes retangulares.

A PCrit foi calculada através do método não linear de acordo com a equação hiperbólica:

$$
t \lim =C T A /(P-P C r i t)
$$

O ajuste foi considerado satisfatório ao apresentar um erro padrão da estimativa (EPE) dos parâmetros, abaixo de $10 \%{ }^{13}$. A opção de uso da equação hiperbólica, em detrimento das formas lineares correlatas ${ }^{14}$, foi embasada matematicamente pela caracterização da potência como a variável independente no tipo de exercício adotado ${ }^{15}$.

$O$ teste retangular adicional realizado na intensidade da PCrit não teve caráter exaustivo e a duração foi estabelecida em 10 minutos de esforço. Os procedimentos para coleta e determinação do $\mathrm{VO}_{2}$ inerente à carga foram os mesmos adotados anteriormente.

Para verificar se a magnitude do $\mathrm{VO}_{2}$, em cargas retangulares no domínio severo, sofre influência do tempo de permanência em exercício, foram selecionados quatro momentos: $120\left(\mathrm{VO}_{2-120}\right), 210$ $\left(\mathrm{VO}_{2-210}\right), 390$ segundos $\left(\mathrm{VO}_{2-390}\right)$ e ao final do exercício $\left(\mathrm{VO}_{2-\mathrm{Fim}}\right) . \mathrm{O} \mathrm{VO}_{2}$ desses períodos foi calculado como a média dos últimos 30 segundos prévios ao ponto temporal estabelecido. Esses valores foram comparados entre si e com os observados durante o teste realizado na intensidade da PCrit. Apenas os dados dos participantes que realizaram os testes na PCrit foram incluídos nessa análise temporal.

\section{Análise Estatística}

Para verificar a normalidade na distribuição dos dados foi aplicado teste de Shapiro Wilk. Os valores de $\mathrm{VO}_{2 \max -\mathrm{INC}}$ e $\mathrm{VO}_{2 \text { max-RET}}$, assim como, a Ppico e as intensidades $\mathrm{L}, \mathrm{M}$ e $\mathrm{P}$ foram comparados através de Anova one-way para medidas repetidas. Para os valores de $\mathrm{VO}_{2 \mathrm{INC}}$ e $\mathrm{VO}_{2 \mathrm{RET}}$, utilizou-se Anova twoway para medidas repetidas, tendo como fatores 0 modo como o exercício foi realizado (incremental ou retangular) e a intensidade ( $L, M, P$ ou $P C$ rit). A análise temporal da magnitude do $\mathrm{VO}_{2}$ ou a comparação do $\mathrm{VO}_{2}$ entre as diferentes intensidades foram realizadas através de Anova one way para medidas repetidas. Análise post hoc de Newman Keuls foi aplicada quando necessário. O nível de significância adotado em todas as análises foi $p<0,05$.

\section{RESULTADOS}

Na tabela 1, são apresentadas: a média das Ppico encontradas no teste incremental, das intensidades aplicadas para determinar a PCrit e dos valores máximos de $\mathrm{VO}_{2}$ observados em cada situação. Não foram encontradas diferenças entre os valores de $\mathrm{VO}_{2 \text { max-INC }}$ e os valores de $\mathrm{VO}_{2 \text { max-RET }}$ observados nos testes retangulares. A Ppico foi igual apenas à intensidade $\mathrm{M}$.

Tabela 1. Média e desvio padrão da potência pico (Ppico) realizada no teste incremental, das intensidades preditivas do modelo da potência crítica (Leve, Média e Pesada) e do $\mathrm{VO}_{2}$ observado para essas intensidades.

\begin{tabular}{ccccc}
\hline & Ppico & Leve & Média & Pesada \\
\hline Intensidade & $255 \pm$ & $220 \pm$ & $246 \pm$ & $288 \pm$ \\
$(\mathrm{W})$ & $49,8^{\mathrm{b}}$ & $35,8 \mathrm{a}^{\mathrm{a}}$ & $38,5^{\mathrm{b}}$ & $40,8^{\mathrm{a}}$ \\
$\mathrm{VO}_{2}\left(\mathrm{l} \cdot \mathrm{min}^{-1}\right)$ & $3,7 \pm 0,5$ & $3,8 \pm 0,6$ & $3,6 \pm 0,5$ & $3,4 \pm 0,6$ \\
\hline
\end{tabular}

a diferente de todas intensidades. ${ }^{\mathrm{b}}$ diferente da Leve e da Pesada, $\mathrm{p}<0,05$

As médias dos tlim nos testes retangulares exaustivos foram $182 \pm 35 \mathrm{~s}, 315 \pm 29 \mathrm{~s}$ e $465 \pm 92 \mathrm{~s}$ para as intensidades $P, M$ e $L$, respectivamente. As estimativas da PCrit (168 $\pm 50,4 \mathrm{~W})$ apresentaram bom ajuste $\left(r^{2}\right.$ entre 0,94 e 1,0). Entretanto, a média da Pcrit foi significativamente menor do que o ponto de compensação respiratória de $205 \pm 35$ W encontrado no teste incremental.

Os valores observados de $\mathrm{VO}_{2 \mathrm{NNC}}$ não apresentaram diferença em relação ao $\mathrm{VO}_{2 \mathrm{RET}}$ nas intensidades $\mathrm{P}, \mathrm{M}$ e PCrit. Entretanto, os valores observados de $\mathrm{VO}_{2 \mathrm{INC}}$ foram menores em comparação ao $\mathrm{VO}_{2 \mathrm{RET}}$ na intensidade L (figura 1).

Devido à amplitude dos tlim encontrados (130 - 632 s), apenas as intensidades L e PCrit participaram de todos os quatro momentos selecionados para a análise temporal. A intensidade $\mathrm{P}$ preencheu o $\mathrm{VO}_{2-120}$ e em seguida o $\mathrm{VO}_{2-\mathrm{Fim}}$, a intensidade $\mathrm{M}$ não participou apenas do momento $\mathrm{VO}_{2-390}$.

Com exceção do momento final, $\mathrm{o} \mathrm{VO}_{2}$ nas intensidades mais baixas foi sempre menor em 
comparação com as cargas mais altas. No momento $\mathrm{VO}_{2 \text {-rim }}$, apenas o valor correspondente à $\mathrm{PCrit}$ continuou menor do que seus pares. Com relação ao $\mathrm{VO}_{2}$, nos diferentes momentos, dentro da própria intensidade, as intensidades $\mathrm{P}$ e $\mathrm{M}$ foram sempre menores em comparação com as medidas seguintes. Entretanto, na intensidade $L$ e na $P C$ rit não houve diferença entre $\circ \mathrm{VO}_{2}$ do momento final e o anterior (figura 2).

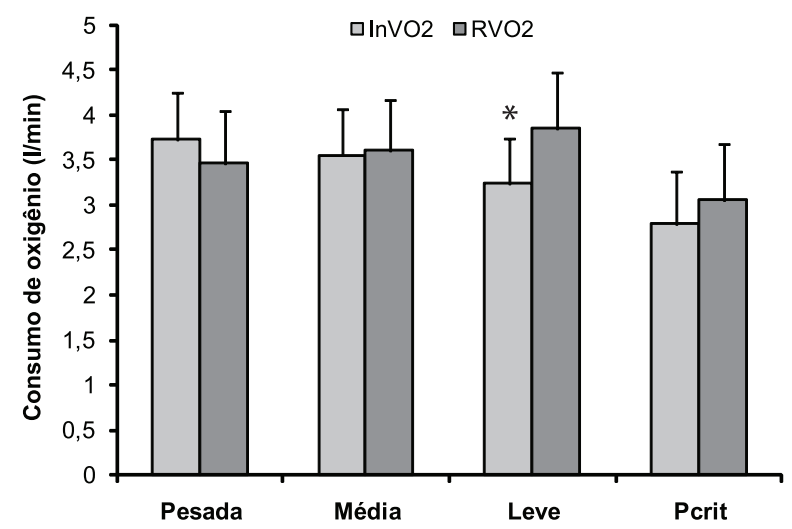

Figura 1. Comparação do $\mathrm{VO}_{2}$ observado no teste incremental $\left(\mathrm{VO}_{2 \mathrm{NC}}\right)$ com o $\mathrm{VO}_{2}$ observado nos testes retangulares $\left(\mathrm{VO}_{2 \mathrm{RET}}\right)$ exaustivos $(n=8)$ e na intensidade da PCrit $(n=5)$. * difere do modo retangular na mesma intensidade, $p<0,05$.

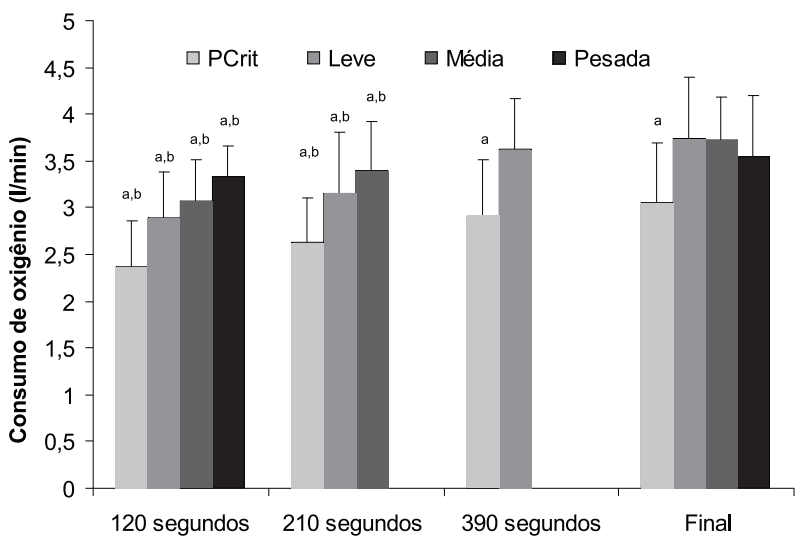

Figura 2. Consumo de oxigênio nos testes retangulares, nos tempos $120,210,390$ segundos e no momento final do exercício. a diferente das outras intensidades dentro do mesmo período. ${ }^{\mathrm{b}}$ diferente do valor observado para a mesma intensidade no momento subseqüente, $p<0,05$.

\section{DISCUSSÃO}

Os testes incrementais possibilitam, com apenas uma visita ao laboratório, a determinação de vários parâmetros da função aeróbia ${ }^{16}$. Contudo, uma preocupação de Day et al. ${ }^{2}$ foi verificar se o $\mathrm{VO}_{2 \max }$, obtido em teste incremental (denominado $\mathrm{VO}_{2 \text { pico }} \mathrm{em}$ seu trabalho), não seria diferente do $\mathrm{VO}_{2 \max }$ observado em testes retangulares máximos. De fato, foi reportado não haver diferença estatística entre os dois valores. Esses resultados confirmam a observação de Hill e
Smith ${ }^{12}$ que, embora não fosse o objetivo do estudo, reportaram igualdade para o $\mathrm{VO}_{2 \max }$ observado em teste incremental e em cargas retangulares exaustivas. Os resultados encontrados no presente estudo também corroboram esses achados. Independente do modo como o exercício foi imposto, o mesmo valor máximo de $\mathrm{VO}_{2}$ foi alcançado para intensidades diferentes. Essa constatação é importante para validar o pressuposto assumido na determinação do $\mathrm{VO}_{2 \mathrm{NC}}$ em intensidades superiores à Ppico.

Estudos envolvidos na estimativa da demanda de $\mathrm{O}_{2}$ para cargas supramáximas ${ }^{3}$, usualmente, utilizam a extrapolação da demanda conhecida em intensidades submáximas. Porém, o intuito do presente trabalho esteve centrado na resposta metabólica real que o organismo oferece mediante a exigência muscular.

Verificamos que o $\mathrm{VO}_{21 \mathrm{NC}}$ subestimou o valor encontrado de $\mathrm{VO}_{2 \mathrm{RET}}$ somente na carga mais leve. Isso confirma a hipótese do presente estudo de que o teste incremental subestima o $\mathrm{VO}_{2}$ real em intensidades mais leves do domínio severo. Adicionalmente, a análise temporal permitiu observar que, em comparação com as intensidades do mesmo domínio, a intensidade $\mathrm{L}$ necessitou de um tempo maior para atingir o $\mathrm{VO}_{2 \max }$. Esse resultado corrobora a hipótese de que, nas intensidades do domínio severo, o $\mathrm{VO}_{2 \max }$ apenas será atingido caso o tempo decorrido de esforço seja suficiente ${ }^{5}$.

A cinética do $\mathrm{VO}_{2}$ em intensidades do domínio intenso é comumente ajustada com modelos exponenciais de dois parâmetros ${ }^{4,6,7}$. Esse comportamento se deve ao surgimento de um componente adicional, de característica mais lenta, que posterga a estabilização e aumenta os valores de $\mathrm{VO}_{2}$ acima dos previstos através de extrapolação, em cargas do domínio moderado 4 .

O curto tempo de duração dos estágios, no teste incremental, em adição ao componente lento, não permitiriam a estabilização do $\mathrm{VO}_{2}$ nas intensidades inerentes ao domínio intenso, de modo que, na carga da PCrit, poder-se-ia esperar uma subestimação do $\mathrm{VO}_{2}$ a partir do $\mathrm{VO}_{2 \mid \mathrm{NC}}$. Porém, não foi observada diferença entre $\circ \mathrm{VO}_{2 \mathrm{NCC}}$ e o $\mathrm{VO}_{2 \mathrm{RET}}$, esse fato exclui uma possível interferência do componente lento na estimativa do $\mathrm{VO}_{2 \mathrm{INC}}$ para essa intensidade. Talvez o estímulo metabólico proveniente das cargas anteriores seja um fator de compensação do sistema nessas condições.

Contrariamente ao domínio intenso, a diferença encontrada entre $\circ \mathrm{VO}_{2 I N C}$ e $\circ \mathrm{VO}_{2 R E T}$ na intensidade $L$ do domínio severo não poderia, ao menos conceitualmente, ser atribuída à ocorrência do componente lento. Pois, foi demonstrado que a cinética do $\mathrm{VO}_{2}$ no domínio severo ajusta-se a um modelo mono-exponencial ${ }^{8}$ e, por conseguinte, não deveria apresentar a manifestação desse fenômeno.

É interessante observar que a influência do tempo foi característica exclusiva do domínio severo. Pois, na intensidade da PCrit, o tempo de esforço foi acima da média em comparação com a intensidade $L$, mas o $\mathrm{VO}_{2 \max }$ não foi alcançado. A PCrit é entendida 
como a intensidade limítrofe entre o domínio intenso e o severo ${ }^{4,17}$. As limitações do presente estudo, como determinar o término da tarefa aos dez minutos e não testar intensidades inferiores à PCrit, são atenuadas ao se assumir o pressuposto de que o alcance do $\mathrm{VO}_{2 \max }$ não é uma característica pertinente ao domínio intenso ${ }^{4}$. Assim, pode-se inferir que para algumas intensidades, dentro do domínio severo, a ocorrência do $\mathrm{VO}_{2 \max }$ apresenta uma interação com o tempo de esforço não observada no domínio intenso e não prevista através de teste incremental.

Adicionalmente, os resultados encontrados corroboram o construto teórico de que na intensidade da PCrit o $\mathrm{VO}_{2 \max }$ não seria atingido ${ }^{9}$. Entretanto, Hirai et al. ${ }^{18}$ observaram valores iguais ao $\mathrm{VO}_{2 \max }$ em corrida realizada até a exaustão, na intensidade da velocidade crítica (similar à PCrit). Talvez um dos motivos capazes de explicar essas diferenças das respostas metabólicas seja a grandeza do erro de estimativa do modelo. As margens de erro aceitáveis são estipuladas para no máximo $10 \%{ }^{13}$. Contudo, as variações diárias de desempenho ${ }^{19}$ poderiam, teoricamente, somar-se aos erros da estimativa. Como a PCrit se encontra em uma zona limítrofe de dois domínios com características diferentes ${ }^{4}$, é possível que ela seja deslocada para um ou outro. Entretanto, são necessários estudos adicionais para elucidar a dissonância entre as respostas metabólicas encontradas através de alguns desenhos experimentais indutivos ${ }^{18,20,21}$ e os pressupostos teóricos da PCrit ${ }^{11,12}$.

\section{CONCLUSÃO}

$\mathrm{O} \mathrm{VO}_{2}$ estimado, a partir de testes incrementais, para intensidades mais leves do domínio severo, subestima o valor observado em testes retangulares.

\section{REFERÊNCIAS BIBLIOGRÁFICAS}

1. Wasserman K, Kessel ALV, Burton GG. Interaction of physiological mechanisms during exercises. J Appl Physiol 1967;22:71-85.

2. Day JR, Rossiter HB, Coats EM, Skasick A, Whipp BJ. The maximally attainable VO2 during exercise in humans: the peak vs. maximum issue. J Appl Physiol 2003;95:1901-1907.

3. Hughson RL, O'leary DD, Betik AC, Hebestreit H. Kinetics of oxygen uptake at the onset of exercise near or above peak oxygen uptake. J Appl Physiol 2000;88:1812-1819.

4. Gaesser GA, Poole DC. The slow component of oxygen uptake kinetics in humans. Exerc Sport Sci Rev 1996;24:35-70.
5. Whipp BJ, Wasserman K. Oxygen uptake kinetics for various intensities of constant-load work. J Appl Physiol 1972;33(3):351-356.

6 . Barstow TJ, Molé PA. Linear and nonlinear characteristics of oxygen uptake kinetics during heavy exercise. J Appl Physiol 1991;63:2253-2261.

7. Barstow TJ. Characterization of $\mathrm{VO}_{2}$ kinetics during heavy exercise. Med Sci Sports Exerc 1994;26:1327-1334.

8. Ozyener F, Rossiter HB, Ward AS, Whipp BJ. Influence of exercise intensity on the on- and off- transient kinetics of pulmonary oxygen uptake in humans. J Physiol 2001;533.3: 891-902.

9. Hill DW, Poole DC, Smith JC. The relationship between power and the time to achieve $\mathrm{VO}_{2 \max }$. Med Sci Sports Exerc 2002;34:709-714.

10 - Taylor HL, Buskirk E, Henschel A. Maximal oxygen intake as objective measure of cardiorespiratory performance. J Appl Physiol 1955;8:73-80.

11. Monod H, Scherrer J. The work capacity of a sinergic muscular group. Ergonomics 1965;8:329-338.

12. Hill DW, Smith JC. Determination of Critical Power by Pulmonary Gas Exchange. Can J Appl Physiol 1999;24:74-86.

13. Hill DW. The critical power concept: A review. Int J Sport Med 1993;16:237-254.

14. Moritani T, Nagata A, Devries HA, Muro M. Critical power as measure of physical work capacity and anaerobic threshold. Ergonomics 1981;24:339-350.

15. Gaesser GA, Carnevale TJ, Garfinkel A, Walter DO, Womack CJ. Estimation of critical power with nonlinear and linear models. Med Sci Sports Exerc 1995;27:14301438.

16. Whipp BJ, Davis JA, Torres F, Wasserman K. A test to determine parameters of aerobic function during exercise. J Appl Physiol 1981;50:217-221.

17. Nakamura FY, Brunetto AF, Hirai DM, Rosseguini BT, Kokubun E. O limiar de esforço percebido (LEP) corresponde à potência crítica e a um indicador de máximo estado estável de consumo de oxigênio. Rev Bras Med Esporte 2005;11(3):197-202.

18. Hirai DM, Brunetto AF, Nakamura FY. Consumo de oxigênio em exercício exaustivo realizado na velocidade crítica e na velocidade associada ao $\mathrm{VO}_{2 \max }$. Rev Educ Física/UEM 2007;18:80-84.

19. Hickey MS, Costill DL, McConell GK, Widrick JJ, Tanaka $\mathrm{H}$. Day to day variation in time trial cycling performance. Int J Sports Med 1992;13(6):467-470.

20. Brickley G, Doust J, Willians CA. Physiological responses during exercise to exhaustion at critical power. Eur J Appl Physiol Occup Physiol 2002;88:146-151.

21. Carter H, Grice Y, Dekerle J, Brickley G, Hammond AJP, Pringle JSM. Effect of prior exercise above and below critical power on exercise to exhaustion. Med Sci Sports Exerc 2005;37(5):775-781.

\section{Endereço para correspondência}

Rodrigo Ferreira de Moura

Departamento de Educação Física

Núcleo de Atividade Física Esporte e Saúde - NAFES

Recebido em 13/08/07

Av.: 24-A, 1515 - Bela Vista

Cep: 13506-900 - Rio Claro - SP, Brasil

E-mail: mourafit@yahoo.com.br 